\title{
Is Your Food Really Safe and Nutritive to Eat? Just Think For a While.....
}

\section{IJCRR}

Section: Healthcare

Sci. Journal Impact

Factor: 6.1 (2018)

ICV: 90.90 (2018)

\section{Debarshi Kar Mahapatra}

Department of Pharmaceutical Chemistry, Dadasaheb Balpande College of Pharmacy, Nagpur 440037, Maharashtra, lndia Email:dkmbsp@gmail.com
There is hardly any individual across the globe that does not have any likings for a particular food preparation to eat. Cheap fast junk food remains a craze among youngsters. But, have we thought about the safety of the food or not, otherwise, this can result in a variety of illnesses according to the type of food and the preparation process. On the other hand, it might be securely concluded that the most hazardous form is the "under-cooked animal products". Sickness as a result of ingestion of natural toxins in food or toxic materials due to contamination or ingestion of living organisms or larvae or their eggs offers in the food; and lastly, adulterated food are the primary causes for frequently occurring difficulties.

Let's focus on the nutritional aspects of food. Low birth weight of $<2.5 \mathrm{Kg}$ occurs in nearly $1 / 3 \mathrm{rd}$ of babies born in India due to anemia, maternal malnutrition, and physical labor during pregnancy. The babies of the mentioned conditions are largely associated with retardation growth and are more prone to certain classes of infections. In early childhood, protein-energy malnutrition occurs where two clinical conditions such as Marasmus and Kwashiorkor have been frequently reported. Intake of poor quantity and quality of food are the chief reasons and this condition prolongs when respiratory infection, frequent diarrhea, and worm infestations are associated. Night blindness results due to Vitamin-A nutritional deficiency which leads to loss of vision. Vitamin-B-complex deficiency offers an increase in multiple disorders based on specific deficiencies as it comprises of numerous constituents. The classic examples include Pellagra (tingling and numbness of hands and feet), Beriberi (reddish ulceration of the tongue), Megaloblastic anemia (neurological deficiency), and Angular stomatitis (small ulceration of the angles of the mouth). Iodine deficiency is prevalent in definite regions of India which causes Goiter whereas, in contrast, Endemic Fluorosis results in skeletal deformities and teeth deformities due to high fluoride levels. Heart disease is contributed to high triglycerides and high cholesterol levels.

Let's focus on the toxins in food. Lathyrism is a crippling nervous disease that affects the lower limbs due to the consumption of a toxic compound present in Lathyrus sativa or 'khesari dal'. It is freely available in definite areas of India and vastly affects the population. Aspergillus flavus, a well-known fungus that grows in food grains such as maize, rice, groundnut, wheat, etc. produces aflatoxin that drastically affects the liver and is susceptible to initiate cancer. Claviceps fusiformis, a fungus that grows in the form of black mass (Ergot) on crops such as rye, wheat, bajra, etc. which causes ergot poisoning on consumption and ultimately leads to gangrene. Epidemic Dropsy results due to the addition of Argemone mexicana seeds to the mustard seeds. This contaminated mustard seeds on consumption lead to non-inflammatory swelling of both legs as well as other symptoms. Fusarium species are the class of fungi that contains toxins which infect food grains. Crotalaria seeds containing certain potent toxins are mixed or contaminated with millets leads to jaundice, liver damage, and fluid accumulation in the abdominal region. This condition is termed as Endemic Ascitis.

Let's focus on the bacteria present in food. Food poisoning occurs on the consumption of farm products (contaminated milk) and poultry products, leads to vomiting, fever, abdominal cramps, and diarrhea due to the presence of specific heat-stable enterotoxins. Salmonella bacteria, Bacillus cereus bacteria, and Staphylococcus bacteria from infected cattle affect the human population. Typhoid and Cholera are the two prominent diseases which results from fecal-oral contamination from food. The heat-sensitive exotoxin from Clostridium botulinum causes Botulism that causes visual problems, weakness, muscle difficulty in speaking, etc. The spore of this microorganism is extensively spread in the dust, soil, contaminated food, and animal.

Let's focus on the worms situated in food. Amoebiasis is caused by consuming the food infected with Entamoeba histolytica that results in mild diarrhea, local ulceration, liver abscess, and ultimately affecting the lungs. Giardiasis is caused by Giardia lamblia that leads to flatulence and mucous diarrhea. Chinese liver fluke is a worm that affects the human liver when inadequately cooked pickled fish (infected with Clonorchis sinensis) is consumed. Similarly, Paragonimus westermani, a lung fluke (worm) causes infection when undercooked crayfish and crabs are ingested. The consumption of undercooked pork causes infection from tapeworm Taenia solium whereas Taenia saginata induces infection on the consumption of undercooked beef. A number of intestinal worms (threadworm, whipworm, hookworms, and roundworm) like Necator americanus, Strongyloides stercoralis, Trichuris trichiura, Ascaris lumbricoides, Ancylostoma duodenale, Enterobius vermicularis, etc. causes numerous GIT-oriented diseases.

Let's focus on the adulterants in food. We people are highly conscious about the powdered spices that are particularly adulterated with sawdust and inferior 'mawa' is employed in preparing the sweets during the Indian festival. Food Adulteration is referred to as a food product that fails to uphold the authorized standards and is incredibly widespread in India. Usually, it is adding up of other materials to a food component for boosting the food quantity that affects the loss of actual quality. Here, new terms will be introduced to the readers that will assist us to emerge from this gloomy picture; Food Additives (addition of flavoring agents, preservatives, and coloring agents for improving texture, appearance, storage properties, and flavor) and Food Fortification (adding up of small quantity nutrients to improve the product quality such as common salt iodization, Vitamin-D to Vanaspati, etc.).

So, it can be well valued that balanced diet foods and food products are an enormous subject covering lots of scientific fields and should in no way be taken carelessly. But every individual must forever be healthy by consuming high quality nourishing food that has been made and served hygienically. 